\title{
Youth Energy Expenditure During Common Free-Living Activities and Treadmill Walking
}

\begin{abstract}
John M. Schuna Jr., Tiago V. Barreira, Daniel S. Hsia, William D. Johnson, and Catrine Tudor-Locke
Background: Energy expenditure (EE) estimates for a broad age range of youth performing a variety of activities are needed. Methods: 106 participants (6-18 years) completed 6 free-living activities (seated rest, movie watching, coloring, stair climbing, basketball dribbling, jumping jacks) and up to 9 treadmill walking bouts ( 13.4 to $120.7 \mathrm{~m} / \mathrm{min} ; 13.4 \mathrm{~m} / \mathrm{min}$ increments). Breath-by-breath oxygen uptake $\left(\mathrm{VO}_{2}\right)$ was measured using the COSMED K4b $\mathrm{b}^{2}$ and $\mathrm{EE}$ was quantified as youth metabolic equivalents $\left(\mathrm{MET}_{\mathrm{y} 1}: \mathrm{VO}_{2} /\right.$ measured resting $\mathrm{VO}_{2}, \mathrm{MET}_{\mathrm{y} 2}: \mathrm{VO}_{2}$ /estimated resting $\mathrm{VO}_{2}$ ). Age trends were evaluated with ANOVA. Results: Seated movie watching produced the lowest mean $\mathrm{MET}_{\mathrm{y} 1}$ (6- to 9-year-olds: $0.94 \pm 0.13$ ) and $\mathrm{MET}_{\mathrm{y} 2}$ values (13- to 15-year-olds: $1.10 \pm 0.19$ ), and jumping jacks produced the highest mean $\mathrm{MET}_{\mathrm{y} 1}$ (13- to 15-year-olds: $6.89 \pm 1.47$ ) and $\mathrm{MET}_{\mathrm{y} 2}$ values (16- to 18-year-olds: 8.61 \pm 2.03 ). Significant age-related variability in $\mathrm{MET}_{\mathrm{y} 1}$ and $\mathrm{MET}_{\mathrm{y} 2}$ were noted for 8 and 2 of the 15 evaluated activities, respectively. Conclusions: Descriptive EE data presented herein will augment the Youth Compendium of Physical Activities.
\end{abstract}

Keywords: sedentary behavior, public health, physical activity, epidemiology

Over 20 years have passed since the introduction of the Compendium of Physical Activities, and the original publication, ${ }^{1}$ in conjunction with subsequent updates, ${ }^{2,3}$ have collectively received more than 5000 citations (Web of Science, accessed December 16, 2015). The compendium provides standardized estimates of energy expenditure (EE) across a broad array of activities, represented in absolute terms as metabolic equivalents (METs). MET values contained within the compendium are applicable to adult populations and typically used to describe the metabolic cost of self-reported behaviors in epidemiological research. ${ }^{1-3}$

More recently, a youth compendium (now referred to as the Youth Compendium of Physical Activities) was introduced to catalog EE estimates for children and adolescents. ${ }^{4}$ At present, there remains a need to expand the assembled activities and age ranges represented within the Youth Compendium of Physical Activities. Therefore, the primary purpose of this study was to provide descriptive data of measured EE in children and adolescents during common freeliving activities and treadmill walking. We also examined the age independence of several candidate EE metrics across the completed activities.

\section{Methods}

\section{Participants}

A convenience sample of 106 children and adolescents (6-18 years) from Louisiana participated in the study. Participants were

Schuna (John.Schuna@oregonstate.edu) is with the School of Biological and Population Health Sciences, Oregon State University. Barreira is with the Dept of Exercise Science, Syracuse University. Hsia is with the Joint Diabetes Endocrinology and Metabolism Program; Johnson is with the Biostatistics and Analysis Core, Pennington Biomedical Research Center, Baton Rouge, LA. Tudor-Locke is with the Dept of Kinesiology, University of Massachusetts Amherst. Schuna, Barreira, and Tudor-Locke are also with the Pennington Biomedical Research Center, Baton Rouge, LA. recruited using e-mail advertisements, health fair presentations, and via word-of-mouth. The Pennington Biomedical Research Center Institutional Review Board approved all study protocols. Before participation, informed parental consent and assent were obtained for 6- to 17-year-old participants, and informed consent was obtained for 18-year-old participants. The study was prospectively registered at ClinicalTrials.gov before enrollment began (NCT01989104).

\section{Activities}

Participants completed laboratory-based free-living activities and ambulatory treadmill bouts while oxygen uptake $\left(\mathrm{VO}_{2}\right)$ was measured. A 4-hour fast (no food or caloric/caffeinated beverages) was required and a 10-minute seated washout period preceded the protocol. Participants then completed 3 seated activities by resting motionless, watching a movie, and coloring in a book. A series of up to 10 ambulatory treadmill bouts ( $0 \%$ grade) followed the seated activities, beginning at $13.4 \mathrm{~m} / \mathrm{min}(0.80 \mathrm{~km} / \mathrm{h})$ and increasing in $13.4 \mathrm{~m} / \mathrm{min}$ increments to a maximum of $134.1 \mathrm{~m} / \mathrm{min}(8.05 \mathrm{~km} / \mathrm{h})$. Treadmill testing was terminated following the bout when the participant naturally began running. Participants then completed simulated stair climbing (stepping up-and-down on a $19.69 \mathrm{~cm}$ step at $88 \mathrm{bpm}$ ), basketball dribbling while ambulating at a self-selected speed (continuous movement around a $12 \mathrm{~m}$ course), and jumping jacks (126 bpm). Each activity lasted 5 minutes and was preceded by a 2-minute washout period, except for the transition following the final treadmill bout where a 5-minute washout period was given to ensure $\mathrm{VO}_{2}$ returned close to resting values before completing the remaining activities.

\section{Measures}

Weight and body fat percentage were measured to the nearest $0.1 \mathrm{~kg}$ and $0.1 \%$, respectively, using an integrated scale and bioelectrical impedance analyzer (Tanita SC-240; Tanita, Tokyo, Japan). Height was measured shoeless to the nearest $0.1 \mathrm{~cm}$ using a stadiometer 
(Harpenden; Holtain, Crosswell, United Kingdom). Body mass index (BMI) was calculated by dividing weight by height squared $\left(\mathrm{kg} / \mathrm{m}^{2}\right)$. Reference data from the Centers for Disease Control and Prevention were used to calculate BMI percentiles. ${ }^{5}$

Respiratory gas concentrations and flow volumes were measured during all activities using the portable COSMED K4b ${ }^{2}$ (COSMED, Rome, Italy). A silicone face mask directed respiratory gases through an external housing where breath-by-breath gas sampling and turbine-based flow volume measurements were taken. Before each testing session, the $\mathrm{K} 4 \mathrm{~b}^{2}$ was calibrated according to the manufacturer's instructions. Measurements from the $\mathrm{K} 4 \mathrm{~b}^{2}$ were transmitted wirelessly and saved to a laptop computer.

Recorded breaths within each minute were averaged to yield a minute-by-minute data file. Absolute and mass-specific $\mathrm{VO}_{2}(\mathrm{~L} / \mathrm{min}$ and $\mathrm{ml} / \mathrm{kg} / \mathrm{min}$, respectively) were calculated. We also computed youth METs (mass-specific $\mathrm{VO}_{2}$ / resting mass-specific $\mathrm{VO}_{2}$ ) as this metric has been shown to provide balance in attenuating the age- and sex-dependence of youth EE estimates across a range of activities. ${ }^{6}$ Youth METs were derived in 2 forms: 1) $\mathrm{MET}_{\mathrm{y} 1}$ : (mass-specific $\mathrm{VO}_{2}$ / measured resting mass-specific $\mathrm{VO}_{2}$ ) and, 2) $\mathrm{MET}_{\mathrm{y} 2}$ : (mass-specific $\mathrm{VO}_{2}$ / estimated resting mass-specific $\mathrm{VO}_{2}$ ). Measured resting $\mathrm{VO}_{2}$ corresponded to the seated rest testing phase while estimated resting $\mathrm{VO}_{2}$ was calculated using the Schofield equation (resting EE converted to mass-specific $\left.\mathrm{VO}_{2}\right){ }^{7}$ The average value from minutes 4 and 5 of each activity was used for analysis. For all seated activities, average values from minutes 4 and 5 were considered steady-state. Steady-state ascertainments among the remaining activities were evaluated by inspecting the variability of breath-by-breath data from minutes 4 and 5. Minutes with mean absolute $\mathrm{VO}_{2}$ variability $<10 \%$ were deemed steady-state. As treadmill walking was one of the specific foci of this study, we eliminated data from treadmill bouts where running occurred.

\section{Data Analyses}

Descriptive statistics were computed to characterize the sample. For each activity, differences in metabolic measures between 4 age categories $(6-9,10-12,13-15$, and 16-18 years) were evaluated using one-way ANOVA. Follow-up pairwise comparisons were conducted using the Tukey-Kramer method. All analyses were performed using $\mathrm{R}$ (version 3.2.2). Due to the large number of hypothesis tests conducted, we conservatively defined statistical significance as $P<.001$.

\section{Results}

Of the 1696 possible activity bouts [ $(106$ participants $\times 6$ free-living activities $)+(106$ participants $\times 10$ treadmill bouts $)]$, we excluded 316 treadmill bouts (participant began running or bout was not completed), 18 active free-living bouts (bout was not completed), and 1 bout during seated rest (K4b $\mathrm{b}^{2}$ malfunction). Of the remaining 1361 bouts, we further excluded data from 152 bouts not meeting our predetermined steady-state criteria. In all, the remaining 1209 bouts available for analysis represented $88.9 \%$ of the original nonmissing observations.

Descriptive characteristics are presented in Table 1 . The mean age was $12.0 \pm 3.7$ years and $58.5 \%, 15.1 \%$, and $26.4 \%$ of the sample were nonoverweight $(\mathrm{BMI}<85$ th percentile), overweight $(85$ th percentile $\leq$ BMI $<95$ th percentile), or obese (BMI $\geq 95$ th percentile), respectively. The sample was racially/ethnically heterogeneous as $37.7 \%, 59.4 \%$, and $2.8 \%$ identified as African-American, Caucasian, or other race/ethnicity, respectively. The sample's BMI and race/ ethnic characteristics were roughly comparable to previously published state-level estimates in Louisiana (2- to 19-year-old obesity prevalence: 29.0\%; race/ethnic distribution-African-American: $32.3 \%$, Caucasian: $64.0 \%$, other race/ethnicity: $3.7 \%$ ). ${ }^{8,9}$

Age-group stratified mean values of absolute $\mathrm{VO}_{2}$, mass-specific $\mathrm{VO}_{2}, \mathrm{MET}_{\mathrm{y} 1}$, and $\mathrm{MET}_{\mathrm{y} 2}$ during the 6 free-living activities are presented in Table 2. Significant age-related differences in absolute $\mathrm{VO}_{2}$ were observed for all activities, except seated rest and coloring, as age tended to be positively associated with absolute $\mathrm{VO}_{2}$. Massspecific $\mathrm{VO}_{2}$ also significantly differed across age groups for most of the free-living activities, with the exception of jumping jacks; however, age was inversely associated with mass-specific $\mathrm{VO}_{2}$. Less variability in $\mathrm{MET}_{\mathrm{y} 1}$ and $\mathrm{MET}_{\mathrm{y} 2}$ values was observed as significant age-related differences were only noted for stair climbing and jumping jacks with $\mathrm{MET}_{\mathrm{y} 1}$ and seated rest and coloring with $\mathrm{MET}_{\mathrm{y} 2}$. Age was positively associated with $\mathrm{MET}_{\mathrm{y} 1}$ values among those free-living activities with noted significant differences; however, there did not appear to be any consistent relationship between age and $\mathrm{MET}_{\mathrm{y} 2}$ values across the tested free-living activities.

Table 1 Descriptive Characteristics

\begin{tabular}{lccccccc}
\hline Stratification & $\mathbf{n}$ & Age (years) & Weight $(\mathbf{k g})$ & Height $(\mathbf{c m})$ & BMI $\left(\mathbf{k g} / \mathbf{m}^{2}\right)$ & BMI percentile & Percent body fat \\
\hline Total & 106 & $12.0 \pm 3.7$ & $53.8 \pm 22.6$ & $152.8 \pm 16.6$ & $22.2 \pm 6.5$ & $67.2 \pm 30.4$ \\
Boys & 53 & $12.0 \pm 3.7$ & $54.4 \pm 22.3$ & $154.5 \pm 18.5$ & $22.0 \pm 5.9$ & $66.9 \pm 32.0$ & $22.3 \pm 11.0$ \\
Girls & 53 & $12.1 \pm 3.6$ & $53.2 \pm 23.0$ & $151.1 \pm 14.5$ & $22.5 \pm 7.1$ & $67.5 \pm 29.0$ & $28.1 \pm 10.0$ \\
6-9 y & $32(16 \mathrm{~B}, 16 \mathrm{G})$ & $7.6 \pm 1.1$ & $34.5 \pm 13.1$ & $132.9 \pm 8.7$ & $19.2 \pm 5.3$ & $66.4 \pm 32.7$ & $24.7 \pm 9.8$ \\
10-12 y & $25(13 \mathrm{~B}, 12 \mathrm{G})$ & $11.0 \pm 0.8$ & $48.9 \pm 12.6$ & $152.0 \pm 8.1$ & $21.1 \pm 4.7$ & $67.8 \pm 31.0$ & $24.7 \pm 9.2$ \\
$13-15$ y & $26(12 \mathrm{~B}, 14 \mathrm{G})$ & $14.0 \pm 0.8$ & $68.0 \pm 17.6$ & $163.6 \pm 8.6$ & $25.3 \pm 5.8$ & $78.7 \pm 21.2$ & $29.3 \pm 10.7$ \\
$16-18$ y & $23(12 \mathrm{~B}, 11 \mathrm{G})$ & $17.0 \pm 0.9$ & $69.7 \pm 24.4$ & $169.0 \pm 8.2$ & $24.4 \pm 8.3$ & $54.6 \pm 31.8$ & $22.2 \pm 13.6$ \\
African- & $40(21 \mathrm{~B}, 19 \mathrm{G})$ & $11.7 \pm 3.7$ & $56.2 \pm 24.6$ & $152.0 \pm 16.0$ & $23.5 \pm 7.2$ & $76.0 \pm 26.7$ & $28.1 \pm 11.4$ \\
American & & & & & & \\
Caucasian & $63(30 \mathrm{~B}, 33 \mathrm{G})$ & $12.3 \pm 3.7$ & $52.7 \pm 21.3$ & $153.5 \pm 17.0$ & $21.7 \pm 6.0$ & $61.8 \pm 32.0$ & $23.6 \pm 10.6$ \\
Other & $3(2 \mathrm{~B}, 1 \mathrm{G})$ & $10.3 \pm 3.5$ & $41.8 \pm 20.6$ & $147.3 \pm 21.5$ & $18.4 \pm 3.3$ & $63.4 \pm 17.0$ & $21.5 \pm 6.2$ \\
\hline
\end{tabular}

Note. Values presented as counts and Mean \pm SD. BMI percentiles for each participant were calculated using reference data from the Centers for Disease Control and Prevention growth charts.

Abbreviations: BMI, body mass index. 


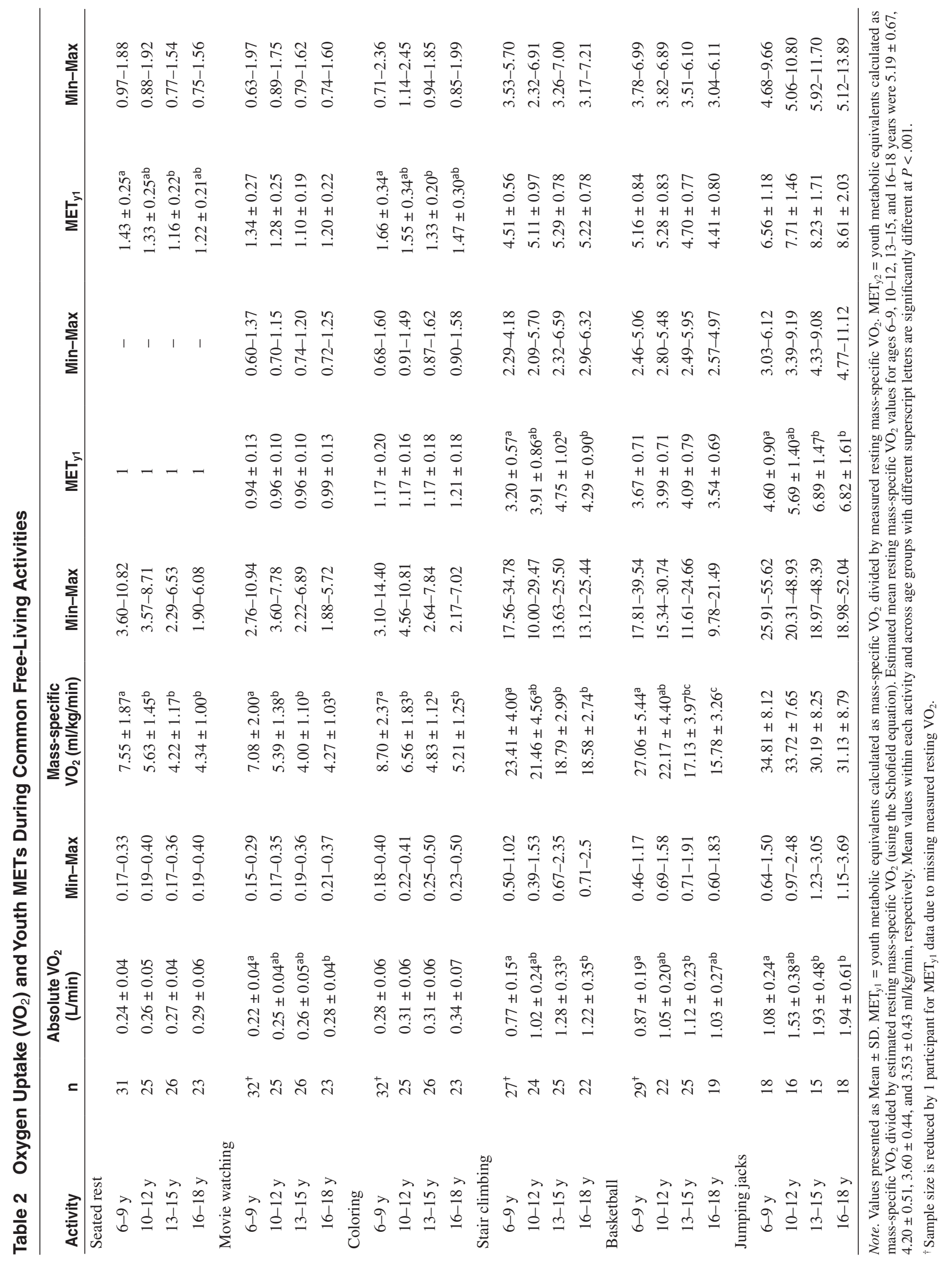




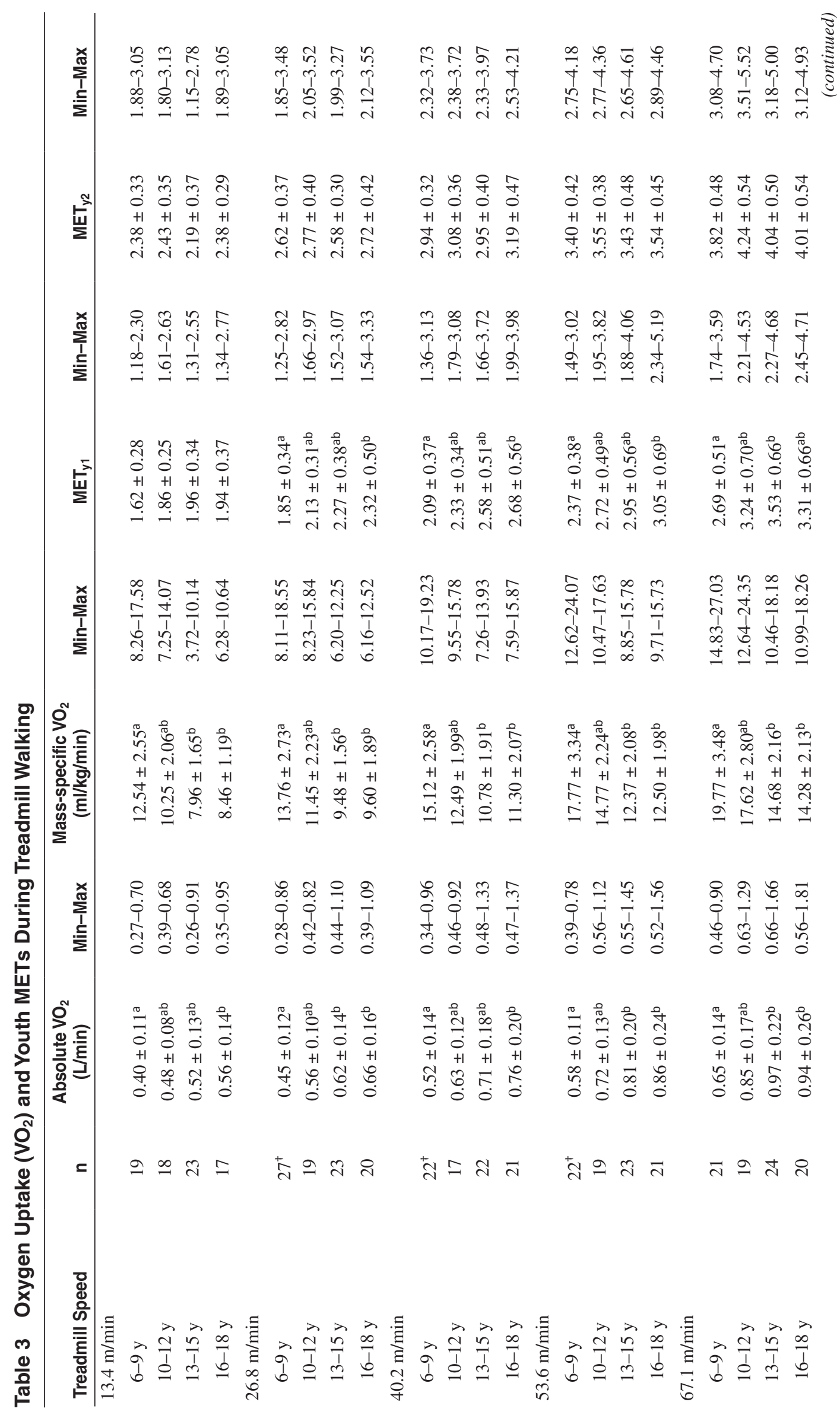




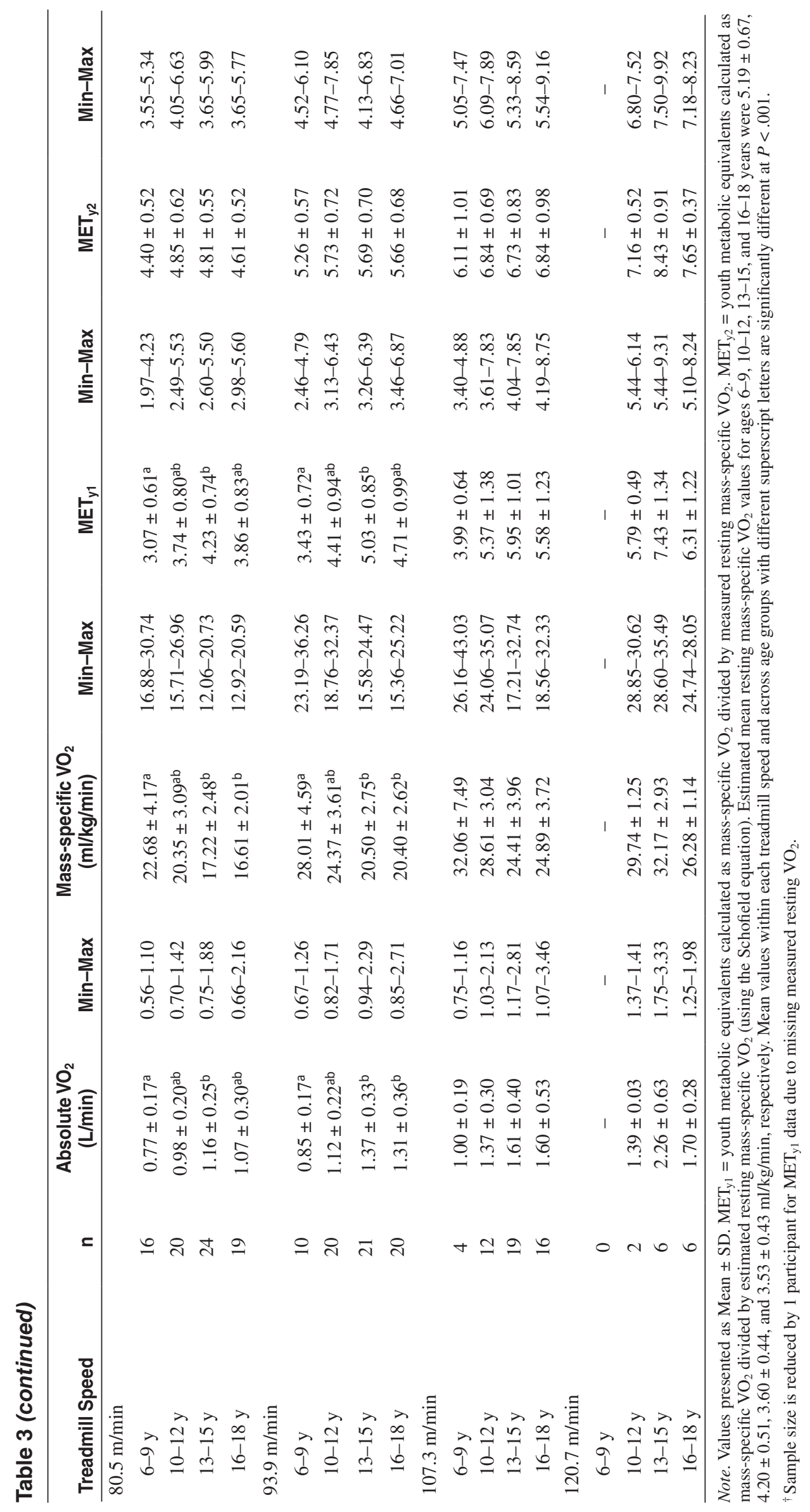


Age-group stratified mean values of absolute $\mathrm{VO}_{2}$, mass-specific $\mathrm{VO}_{2}, \mathrm{MET}_{\mathrm{y} 1}$, and $\mathrm{MET}_{\mathrm{y} 2}$ during treadmill walking are presented in Table 3. All participants that attempted the tenth treadmill bout $(134.1 \mathrm{~m} / \mathrm{min}$ ) began running at this speed; therefore, we restrict our presentation to only those 9 speeds $\leq 120.7 \mathrm{~m} / \mathrm{min}$. Absolute $\mathrm{VO}_{2}$ significantly differed across age groups at all speeds between 13.4 and $93.9 \mathrm{~m} / \mathrm{min}$, as age tended to be positively associated with absolute $\mathrm{VO}_{2}$. Significant age-related differences were also noted for mass-specific $\mathrm{VO}_{2}$ at all speeds between 13.4 and $93.9 \mathrm{~m} / \mathrm{min}$. However, age was inversely associated with mass-specific $\mathrm{VO}_{2}$. Significant differences in $\mathrm{MET}_{\mathrm{y} 1}$ values between age groups were observed at all speeds between 26.8 and $93.9 \mathrm{~m} / \mathrm{min}$, as age tended to demonstrate a positive relationship with $\mathrm{MET}_{\mathrm{y} 1}$. No significant age-related differences in $\mathrm{MET}_{\mathrm{y} 2}$ values were noted across any of the evaluated speeds.

\section{Discussion}

The descriptive data we have presented are novel, and we know of no single study which has reported children and adolescents' steady-state EE estimates across the complete set of walking speeds evaluated herein. Moreover, no published studies have reported steady-state EE estimates among children and adolescents 6 to 18 years of age for the specific set of common free-living activities we evaluated. In light of these observations, our comparative discourse is limited. However, these data serve as a needed addition to previous assemblages of children and adolescents' measured EE during various sedentary and physical activities. ${ }^{4}$

Of the candidate EE metrics evaluated herein, the $\mathrm{MET}_{\mathrm{y} 2}$ appeared to be least associated with age, as age-related differences were only observed among 2 of the study's 15 activities. This finding is consistent with previous work by McMurray et $\mathrm{al}^{6}$ which demonstrated that the $\mathrm{MET}_{\mathrm{y} 2}$ (using estimated resting $\mathrm{VO}_{2}$ as the denominator) tended to be less associated with age and sex across a range of activities than absolute, mass-specific, or net $\mathrm{VO}_{2}$ measures. Interestingly, $\mathrm{MET}_{\mathrm{y} 1}$ demonstrated greater variability across age groups than did $\mathrm{MET}_{\mathrm{y} 2}$, which is likely attributable to the greater age-related variation in directly measured resting $\mathrm{VO}_{2}$ (seated rest: 1.90 to $10.82 \mathrm{ml} / \mathrm{kg} / \mathrm{min}$ ) used in $\mathrm{MET}_{\mathrm{y} 1}$ calculations than in estimated resting $\mathrm{VO}_{2}$ (2.54 to $6.39 \mathrm{ml} / \mathrm{kg} / \mathrm{min}$ ) used in $\mathrm{MET}_{\mathrm{y} 2}$ calculations. Further research is needed to better understand how the formulation of youth METs influences the metric's relationship with children and adolescents' age, sex, body composition, and other physical characteristics.

We have presented estimates of measured steady-state EE during 15 different activities from a diverse sample of youth.
The assembled data herein will inform future pediatric-related epidemiological research and augment the Youth Compendium of Physical Activities.

\section{Acknowledgments}

The authors thank Nancy Rajapho, Melissa Lupo, Stefany Achee, and Courtney Hancock for their assistance with this project. This work was supported by a grant from the Eunice-Kennedy Shriver National Institute of Child Health and Human Development (NIH-NICHD 1R21HD07380701A1). Dr. Johnson also received partial support from NIH-NIGMS (1 U54 GM104940) which funds the Louisiana Clinical and Translational Science Center. The authors declare they have no competing interests.

\section{References}

1. Ainsworth BE, Haskell WL, Leon AS, et al. Compendium of physical activities: classification of energy costs of human physical activities. Med Sci Sports Exerc. 1993;25(1):71-80. PubMed doi:10.1249/00005768-199301000-00011

2. Ainsworth BE, Haskell WL, Whitt MC, et al. Compendium of physical activities: an update of activity codes and MET intensities. Med Sci Sports Exerc. 2000;32(9, Suppl):S498-S504. PubMed doi:10.1097/00005768-200009001-00009

3. Ainsworth BE, Haskell WL, Herrmann SD, et al. 2011 Compendium of Physical Activities: a second update of codes and MET values. Med Sci Sports Exerc. 2011;43(8):1575-1581. PubMed doi:10.1249/ MSS.0b013e31821ece12

4. Ridley K, Ainsworth BE, Olds TS. Development of a compendium of energy expenditures for youth. Int J Behav Nutr Phys Act. 2008;5:45. PubMed doi:10.1186/1479-5868-5-45

5. Kuczmarski RJ, Ogden CL, Grummer-Strawn LM, et al. CDC Growth Charts: United States. Adv Data. 2000; (314):1-27. PubMed

6. McMurray RG, Butte NF, Crouter SE, et al. Exploring metrics to express energy expenditure of physical activity in youth. PLoS One. 2015;10(6):e0130869. PubMed doi:10.1371/journal.pone.0130869

7. Schofield WN. Predicting basal metabolic rate, new standards and review of previous work. Hum Nutr Clin Nutr. 1985;39(Suppl 1):5-41. PubMed

8. U.S. Census Bureau Population Division. Annual Estimates of the Resident Population by Sex, Race, and Hispanic Origin for the United States, States, and Counties: April 1, 2010 to July 1, 2014. Suitland, MD: U.S. Census Bureau; 2015.

9. State of Louisiana Department of Health and Hospitals. Obesity. Baton Rouge, LA: Department of Health and Hospitals; 2015. 